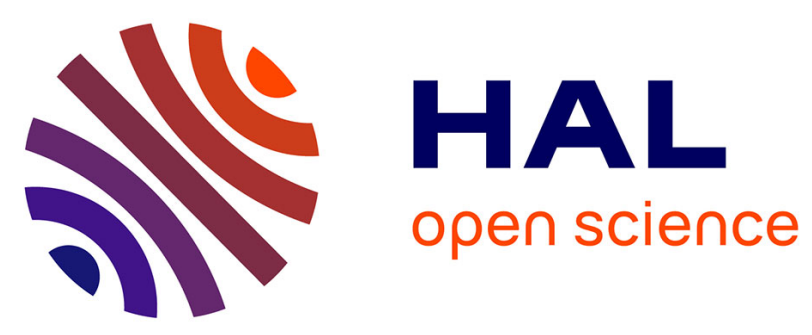

\title{
Meropenem monotherapy is as effective as and safer than imipenem to treat brain abscesses
}

\author{
G. Martin-Canal, A. Saavedra, J.M. Asensi, T. Suarez-Zarracina, A.
}

Rodriguez-Guardado, E. Bustillo, J. Fierer, J.A. Carton, J. Collazos, V.

Asensi

\section{To cite this version:}

G. Martin-Canal, A. Saavedra, J.M. Asensi, T. Suarez-Zarracina, A. Rodriguez-Guardado, et al.. Meropenem monotherapy is as effective as and safer than imipenem to treat brain abscesses. International Journal of Antimicrobial Agents, 2010, 35 (3), pp.301. 10.1016/j.ijantimicag.2009.11.012 . hal-00556386

\section{HAL Id: hal-00556386 https://hal.science/hal-00556386}

Submitted on 16 Jan 2011

HAL is a multi-disciplinary open access archive for the deposit and dissemination of scientific research documents, whether they are published or not. The documents may come from teaching and research institutions in France or abroad, or from public or private research centers.
L'archive ouverte pluridisciplinaire HAL, est destinée au dépôt et à la diffusion de documents scientifiques de niveau recherche, publiés ou non, émanant des établissements d'enseignement et de recherche français ou étrangers, des laboratoires publics ou privés. 


\section{Accepted Manuscript}

Title: Meropenem monotherapy is as effective as and safer than imipenem to treat brain abscesses ${ }^{1}$

Authors: G. Martin-Canal, A. Saavedra, J.M. Asensi, T. Suarez-Zarracina, A. Rodriguez-Guardado, E. Bustillo, J. Fierer, J.A. Carton, J. Collazos, V. Asensi

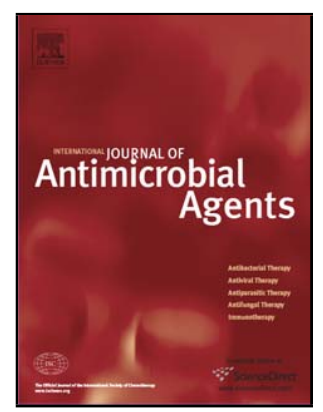

PII: S0924-8579(09)00546-9

DOI:

Reference: doi:10.1016/j.ijantimicag.2009.11.012

ANTAGE 3190

To appear in: International Journal of Antimicrobial Agents

Received date: $\quad$ 25-7-2009

Revised date: $\quad 20-11-2009$

Accepted date: $\quad$ 25-11-2009

Please cite this article as: Martin-Canal G, Saavedra A, Asensi JM, SuarezZarracina T, Rodriguez-Guardado A, Bustillo E, Fierer J, Carton JA, Collazos J, Asensi V, Meropenem monotherapy is as effective as and safer than imipenem to treat brain abscesses 1, International Journal of Antimicrobial Agents (2008), doi:10.1016/j.ijantimicag.2009.11.012

This is a PDF file of an unedited manuscript that has been accepted for publication. As a service to our customers we are providing this early version of the manuscript. The manuscript will undergo copyediting, typesetting, and review of the resulting proof before it is published in its final form. Please note that during the production process errors may be discovered which could affect the content, and all legal disclaimers that apply to the journal pertain. 


\section{Meropenem monotherapy is as effective as and safer than imipenem to treat brain abscesses}

G. Martin-Canal ${ }^{\text {a }}$, A. Saavedra ${ }^{\text {a }}$, J.M. Asensi ${ }^{\text {b }}$, T. Suarez-Zarracina ${ }^{\text {a }}$, A. Rodriguez-Guardado ${ }^{a}$, E. Bustillo ${ }^{\text {c }}$, J. Fierer ${ }^{\text {d, J.A. Carton }}{ }^{\text {a }}$, J. Collazos ${ }^{\text {e }}$, V. Asensi $^{\mathrm{a}, *}$

${ }^{a}$ Department of Infectious Diseases, Hospital Universitario Central de Asturias, Oviedo University School of Medicine, Celestino Villamil s/n, 33006 Oviedo, Spain

${ }^{\mathrm{b}}$ Department of Neurology, Hospital Universitario Central de Asturias, Oviedo University School of Medicine, Oviedo, Spain

${ }^{c}$ Department of Statistics, Hospital Universitario Central de Asturias, Oviedo University School of Medicine, Oviedo, Spain

d Infectious Diseases Section, VAMC and University of California, San Diego, CA, USA

e Infectious Diseases Unit, Hospital de Galdacano, Galdacano, Spain

\section{ARTICLE INFO}

Article history:

Received 25 July 2009

Accepted 25 November 2009

Keywords: 
Brain abscess

Carbapenems

Meropenem

Seizures

Therapy

Neurosurgery

* Corresponding author. Tel.: +34985108000 x38177; fax: +34 985106158 .

E-mail address: vasensia@medynet.com (V. Asensi).

* This work was presented in part at the 48th Interscience Conference on

Antimicrobial Agents and Chemotherapy (ICAAC), 25-28 October 2008, Washington, DC (poster \#L-616). 


\section{ABSTRACT}

The efficacy of carbapenems versus cefotaxime (8 g/day) + metronidazole (1.5$2 \mathrm{~g} /$ day) [combined standard chemotherapy (CSC)] for the treatment of brain abscess was compared. Fifty-nine adult patients with brain abscesses received either imipenem or meropenem (3-4 g/day) or CSC for a mean of 5 weeks, in addition to neurosurgery in most cases. Cure was obtained in $84.7 \%$ of cases; $42 / 47$ (89.4\%) on carbapenems [18/22 (81.8\%) on imipenem versus 24/25 (96.0\%) on meropenem] and 8/12 (66.7\%) on CSC $(P=0.06)$. Seven patients with multiple abscesses were treated with imipenem (1 died; cure rate $85.7 \%$ ), five with meropenem (all survived; cure rate 100\%) and five with CSC (2 died; cure rate $60 \%)(P<0.4)$. Neurosurgery was performed in $43 / 59$ cases $(72.9 \%)$; $17(77.3 \%)$ in the imipenem group, $21(84.0 \%)$ in the meropenem group and 5 $(41.7 \%)$ in the CSC group $(P=0.02)$. There was no significant difference in the rate of relapse requiring re-intervention. Treatment with meropenem was associated with a lower mortality than CSC $(P=0.026)$. Seizures were observed only with carbapenems [8/22 (36.4\%) for imipenem versus 2/25 $(8.0 \%)$ for meropenem; $P=0.03]$. Carbapenems were more effective than CSC for treatment of brain abscesses. Because meropenem induced significantly fewer seizures than imipenem with at least the same clinical efficacy, the former appears to be a better choice to treat this infection. 


\section{Introduction}

Brain abscess is an uncommon infection of the central nervous system with a high morbidity and mortality. It is due primarily to direct spread from an infected paranasal sinus or ear, complication of a neurosurgical procedure or, more rarely, haematogenous dissemination, mostly from the lung and heart [1-3]. It is usually caused by a mixture of microorganisms, with a high proportion of cases due to obligate anaerobes. Broad-spectrum $\beta$-lactam antibiotics, and nowadays the combination of cefotaxime + metronidazole, are the standard therapy for empirical treatment of brain abscess, in addition to neurosurgery $[4,5]$. Carbapenems penetrate well into the cerebrospinal fluid (CSF) and thus are a potential alternative to cephalosporins + metronidazole to treat brain abscesses [6-9]. However, some reports of imipenem-induced seizures, although infrequent, have limited its generalised use $[10,11]$. Meropenem has shown a much lower convulsive activity than imipenem while retaining the same broad antimicrobial spectrum [12-14].

In this 11-year-old retrospective, non-randomised study, we compared the efficacy and safety of intravenous (i.v.) cefotaxime + metronidazole [combined standard chemotherapy (CSC)] with i.v. meropenem or imipenem monotherapy in addition to neurosurgery in the treatment of brain abscesses in adult patients admitted to the Hospital Universitario Central de Asturias (HUCA), Oviedo, Spain. 


\section{Patients and methods}

Medical records from HUCA from January 1996 to December 2007 were retrospectively reviewed to identify cases of brain abscess. HUCA is a 1500bed tertiary university hospital in northwestern Spain providing neurosurgical coverage to a population of 1100000 people.

The diagnosis of brain abscess was made by microbiological, histological, radiographic, surgical and clinical findings as described previously $[2,8]$. Brain abscess was diagnosed in 59 patients; surgically and histologically in 43 patients (40 of whom had positive microbiological cultures) and by compatible clinical picture and computed tomography $(\mathrm{CT})$ or magnetic resonance imaging (MRI) findings in 16 patients who did not undergo neurosurgery. The mean incidence of brain abscess was of 4.9 cases/year. Forty-five patients were men and fourteen were women. The age range was $14-80$ years (mean age $45.9 \pm$ 18.0 years). Patients with the following conditions were excluded from the study: (i) those who were immunocompromised or who tested positive for human immunodeficiency virus (HIV); (ii) those with subdural and epidural abscesses; and (iii) those with fungal, protozoal or mycobacterial abscesses.

Patients received different antimicrobial treatments in a non-randomised manner according to the preference of the infectious diseases consultant. From 2000 onwards, most of the patients were treated with a carbapenem in addition to neurosurgery. Antibiotics were usually started at the time of diagnosis of 
brain abscess, always before neurosurgery, a procedure that could be delayed up to 6 weeks from diagnosis (mean delay 10.5 days; range $0-41$ days).

For the purpose of the study, patients were divided into three groups: Group 1 comprised 12 patients who received CSC (cefotaxime + metronidazole); Group 2 comprised 22 patients who received i.v. imipenem $1 \mathrm{~g} / 6-8 \mathrm{~h}$; and Group 3 comprised 25 patients treated with i.v. meropenem $1 \mathrm{~g} / 6-8 \mathrm{~h}$. No antiepileptic prophylactic therapy was administered prior to or simultaneously with the antibiotics. Treatments lasted a mean of $35.3 \pm 16.3$ days (range 16-84 days).

Pus from brain abscesses obtained by needle aspiration or surgical debridement in 43 patients was sent in capped syringes to the microbiology laboratory for Gram stain and aerobic and anaerobic cultures. Blood cultures were obtained from all the patients and CSF cultures from 15 patients.

\subsection{Statistical analysis}

Qualitative variables were compared by $\chi^{2}$ or Fisher's exact tests as appropriate. The Gaussian distribution of continuous variables was tested with the Kolmogorov-Smirnov test, and the Student's $t$-test or Mann-Whitney U-test was used for comparison of these variables accordingly. One-way analysis of variance (ANOVA), with Scheffé post-hoc test, was used for comparison of more than two groups. The independent association of diverse variables with patient survival was assessed by a Cox proportional hazard model. A $P$-value 
$<0.05$ value for a two-tailed test was considered statistically significant. SPSS

v.15.0 software (SPSS Inc., Chicago, IL) was used for statistical analysis.

\section{Results}

\subsection{Patients}

The characteristics of the patients in the three therapy groups are presented in

Table 1. Carbapenems were used to treat brain abscesses after 2000 at HUCA and the annual incidence of the infection was fairly steady throughout the review period, therefore more than twice as many patients were treated with carbapenems than with CSC. In addition, owing to the non-randomised design of the study, patients in the CSC group were not well matched with those receiving carbapenems. In fact, patients receiving CSC were older, more often presented a Glasgow coma scale (GCS) score $<7$ and more often had multiple abscesses. Patients receiving the two carbapenems were acceptably well matched with regard to the clinical severity of the illness at hospital admission (GCS score, motor weakness, intracranial hypertension), although patients given imipenem were younger. All patients groups were well matched with regard to basic blood and serum determinations such as haemoglobin, total leukocyte count, differential count, platelet count, serum glucose, urea, creatinine and electrolytes $(P=0.1-0.9)$. All antibiotics were administered in a timely manner and at appropriate dosages. 


\subsection{Number of brain abscesses}

Abscesses were single in 42 patients (71.2\%) and multiple in 17 patients $(28.8 \%)$.

\subsection{Microbiology and source of entry of the brain abscesses}

Positive brain pus cultures were obtained in 27 (62.8\%) of 43 cases, positive blood cultures in 15 (25.4\%) of 59 cases and positive CSF cultures in 10 $(66.7 \%)$ of 15 cases. Eleven patients had positive cultures from more than one source. A microbiological diagnosis was based on blood alone in 11 cases (18.6\%) and on CSF alone in another 2 cases (3.4\%). Anaerobes were isolated in $14(23.7 \%)$ of 59 individuals and they were the most frequent isolates, followed by Gram-positive cocci (12/59; 20.3\%), non-anaerobic Gram-negative rods $(11 / 59 ; 18.6 \%)$ and mixed flora $(3 / 59 ; 5.1 \%)$; in 19 patients $(32.2 \%)$ no microorganisms were obtained from the brain pus or other cultures.

Many of the brain abscesses in this series were secondary to neurosurgical procedures $(17 / 59 ; 28.8 \%)$ or head trauma $(2 / 59 ; 3.4 \%)$, followed by those due to ear, nose and throat infections $(14 / 59 ; 23.7 \%)$. Haematogenous brain abscesses were uncommon ( $3 / 59 ; 5.1 \%)$; others were due to other causes $(7 / 59 ; 11.9 \%)$ or were of unknown origin $(16 / 59 ; 27.1 \%)$. 


\subsection{Clinical outcomes}

\subsubsection{Cure}

Patients remained in hospital on i.v. therapy for a mean of $36.5 \pm 22.8$ days (range 5-92 days). Overall, cure was achieved in 50/59 cases (84.7\%), whereas 9 patients (15.3\%) died, mostly due to cranial hypertension and other complications of brain abscess. Four of the patients who died did not undergo neurosurgery. A higher (not statistically significant) cure rate was observed in the carbapenem groups compared with CSC $(89.4 \%$ of patients on carbapenems compared with $66.6 \%$ in the CSC group; $P=0.06$ ). Comparing the two carbapenems, meropenem showed a non-significant slightly greater efficacy than imipenem $(96.0 \%$ cured vs. $81.8 \% ; P=0.17)$. Meropenem was significantly more effective than CSC [24/25 patients (96.0\%) receiving meropenem cured vs. $8 / 12(66.7 \%)$ receiving CSC; $\chi^{2}=3.72$, odds ratio $(\mathrm{OR})=$ 12.0, 95\% confidence interval $(\mathrm{Cl}) 0.98-329.7 ; P=0.03$ by Fisher's exact test] (Table 1). Among the patients with multiple abscesses, seven received imipenem (one died; cure rate $85.7 \%$ ), five received meropenem (all survived; cure rate $100 \%)$ and five received CSC (2 died; cure rate $60 \%)(P<0.4$ for all the comparisons).

Among the different epidemiological, bacteriological, clinical and surgical variables studied, only the GCS score was significantly associated with death ( $P$ $=0.001)$. 


\subsubsection{Neurosurgery}

Neurosurgery was performed in $43 / 59$ patients (72.9\%); $17 / 22$ (77.3\%) on imipenem, $21 / 25(84.0 \%)$ on meropenem and $5 / 12(41.7 \%)$ on CSC $(P=0.02)$. Sixteen patients did not undergo neurosurgery; $1 / 9(11.1 \%)$ of those on carbapenems died and 3/7 (42.9\%) on CSC died ( $P=0.26$ comparing mortality rates of those who did not undergo neurosurgery in the two treatment groups).

Among the 43 patients who underwent neurosurgery, 20 (46.5\%) underwent craniotomy and surgical drainage of the brain abscess, 17 (39.5\%) underwent needle aspiration of the abscess through a burr hole and 6 (14.0\%) had an Ommaya reservoir placed to relieve elevated intracranial hypertension.

\subsubsection{Relapse}

In total, $12(20.3 \%)$ of the 59 patients relapsed requiring re-intervention; 7/22 (31.8\%) on imipenem, $3 / 25(12.0 \%)$ on meropenem $(P=0.1)$ and $2 / 12(16.7 \%)$ on CSC $(P<0.5)$. The second surgical procedure was a new abscess puncture in all but three patients. Only four (33.3\%) of the patients who underwent a second surgery had multiple abscesses.

\subsection{Seizures}

Seizures were observed only in those patients treated with carbapenems $(10 / 47 ; 21.3 \%)$ and not in those on CSC $(P=0.1)$. There were significant differences when the three therapeutic groups were considered altogether $(P=$ 
0.008). However, if imipenem is compared with meropenem, the former induced significantly more seizures [8/22 (36.4\%) for imipenem vs. 2/25 (8.0\%) for meropenem; $\chi^{2}=4.05 ; \mathrm{OR}=6.57,95 \% \mathrm{Cl} 1.04-52.8 ; P=0.03$ by Fisher's exact test]. No other important side effects of antibiotics were reported in the medical charts of patients.

\subsection{Multivariate analysis}

A Cox multivariate proportional hazard model was used to asses the independent association of different variables with death. The model included variables that were found to be related to patient survival on univariate analysis or that could be clinically relevant to this outcome, such as GCS score, antibiotic treatment and the presence or absence of cranial hypertension and surgical treatment. A GCS score $<7$ [hazard ratio $(\mathrm{HR})=26.3,95 \% \mathrm{Cl} 2.2-$ 333.3; $P=0.01)$ and treatment with cefotaxime + metronidazole (CSC) compared with meropenem $(\mathrm{HR}=0.046,95 \% \mathrm{Cl} 0.003-0.692 ; P=0.026)$ were the only variables found to be significantly associated with death (Fig. 1), whereas imipenem treatment $(P=0.13)$, surgical treatment $(P=0.58)$ and cranial hypertension $(P=0.86)$ were not significantly related to this outcome.

\section{Discussion}

Carbapenem monotherapy proved to be an effective alternative to the standard combination of i.v. cefotaxime + metronidazole (CSC) when used in conjunction with neurosurgery to treat bacterial brain abscesses in this study. Meropenem 
showed significantly greater efficacy in univariate analyses than CSC and was independently associated with a favourable outcome by Cox regression. Imipenem-treated patients had the highest incidence of seizures. These observations might make meropenem the best choice for empirical treatment of pyogenic brain abscesses.

This is the largest study of carbapenems for the treatment of brain abscess ever published in the world literature. However, the non-randomised design of the study might have introduced a bias to the results and forces us to be very cautious in interpreting the results. Patients receiving CSC were older, had lower GCS scores, more often had multiple abscesses, less frequently underwent neurosurgery and were admitted before 2000. All these factors, alone or taken together, have been demonstrated to indicate a worse prognosis of brain abscesses in other studies and could work in favour of carbapenems.

Meropenem and imipenem penetrate into the CSF and show excellent antimicrobial activity in meningitis caused by different microorganisms [6$10,13]$. Imipenem has been shown to be present in high concentrations in brain pus and, although not reported so far, meropenem should behave similarly $[9,12,13]$. Both drugs should be present at levels that are well above the minimum inhibitory concentration for $90 \%$ of microorganisms $\left(\mathrm{MIC}_{90}\right)$ for isolates from brain abscesses in this study, mostly obligate anaerobes and Grampositive cocci. 
Overall mortality in this study (15.3\%) was similar to that reported in our previous work and in larger series [2,8]. Jansson et al. [5] reported a much lower short-term abscess-related mortality (4.5\%) and lower relapse rate (8.3\%) with i.v. cefotaxime + metronidazole. However, they observed a 6-month mortality of $12 \%$, similar to ours, and $60 \%$ of patients had adverse reactions, including a seizures rate at the end of therapy of $11 \%$. No seizures were observed in our patients treated with i.v. cefotaxime + metronidazole at the same dose used by Jansson et al. [5].

Meropenem has been shown to be less epileptogenic than imipenem, with a seizure incidence as low as $0.07 \%$ in infections other than meningitis [13]. The convulsive activity of $\beta$-lactams is related to inhibition of $\left[{ }^{3} \mathrm{H}\right]$-muscimol binding to the $\mathrm{GABA}_{\mathrm{A}}$ receptor in brain tissue. In vitro assays and clinical trials have shown that meropenem induced a much lower seizure activity than imipenem, although it was higher than other newer carbapenems such as doripenem and biapenem [14,15]. This study confirms the lower epileptogenic activity of meropenem compared with imipenem even in patients with a high risk of seizures such as brain abscesses with subsequent neurosurgery.

In many countries, i.v. metronidazole is not available and therefore meropenem could be an alternative. However, the cost of 5 weeks of i.v. therapy has to be considered by the clinician and hospital pharmacist because meropenem is the most expensive regimen ( $€ 5177$ for i.v. meropenem, $€ 4900$ for i.v. imipenem and $€ 1639$ for the combination i.v. cefotaxime + metronidazole in Spain). 
We conclude that carbapenem monotherapy was more effective than CSC to treat brain abscesses, in addition to neurosurgery, and produced similar relapses. Meropenem, although the most expensive regimen, induced fewer seizures with slightly better clinical efficacy than imipenem and so may prove to be a better choice to treat this neurological infection.

\section{Funding}

None.

\section{Competing interests}

None declared.

\section{Ethical approval}

Not required. 


\section{References}

[1] Mathisen GE, Johnson JP. Brain abscess. Clin Infect Dis 1997;25:763-9.

[2] Tseng JH, Tseng MY. Brain abscess in 142 patients: factors influencing outcome and mortality. Surg Neurol 2006;65:557-62.

[3] Carpenter J, Stapleton S, Holliman R. Retrospective analysis of 49 cases of brain abscess and review of the literature. Eur $\mathrm{J}$ Clin Microbiol Infect Dis $2007 ; 26: 1-11$

[4] Sjölin J, Lilja A, Eriksson N, Arneborn P, Cars O. Treatment of brain abscess with cefotaxime and metronidazole: prospective study on 15 consecutive patients. Clin Infect Dis 1993;17:857-63.

[5] Jansson AK, Enblad P, Sjölin J. Efficacy and safety of cefotaxime in combination with metronidazole for empirical treatment of brain abscess in clinical practice: a retrospective study of 66 consecutive cases. Eur $\mathrm{J}$ Clin Microbiol Infect Dis 2004;23:7-14.

[6] Carton JA, Perez F, Maradona JA, Mendez FJ. Successful treatment of recurrent cerebral empyema and brain abscesses with imipenem. Eur J Clin Microbiol 1987;6:578-80.

[7] Meis JF, Groot-Loonen J, Hoogkamp-Korstanje JA. A brain abscess due to multiple-resistant Enterobacter cloacae successfully treated with meropenem. Clin Infect Dis 1995;20:1567.

[8] Asensi V, Carton JA, Maradona JA, Asensi JM, Pérez F, Redondo P, et al. Imipenem therapy of brain abscess. Eur J Clin Microbiol Infect Dis 1996;15:653-7. 
[9] Modai J, Vittecoq D, Decazes JM, Meulemans A. Penetration of imipenem and cilastatin into cerebrospinal fluid of patients with bacterial meningitis. $\mathrm{J}$ Antimicrob Chemother 1985;16:751-5.

[10] Asensi V, Carton JA, Maradona JA, Asensi JM, Pérez F, Redondo P, et al. Therapy of brain abscess with imipenem-a safe therapeutic choice? $\mathrm{J}$ Antimicrob Chemother 1996;37:200-3.

[11] Calandra G, Lydick E, Carrigan J, Weiss L, Guess H. Factors predisposing to seizures in seriously ill infected patients receiving antibiotics: experience with imipenem-cilastatin. Am J Med 1988;84:911-8.

[12] Linden P. Safety profile of meropenem: an updated review of over 6,000 patients treated with meropenem. Drug Saf 2007;30:657-68.

[13] Baldwin CM, Lyseng-Williamson KA, Keam SJ. Meropenem: a review of its use in the treatment of serious bacterial infections. Drugs 2008;68:80338.

[14] Day IP, Goudie J, Nishiki K, Williams PD. Correlation between in vitro and in vivo models of proconvulsive activity with the carbapenem antibiotics, biapenem, imipenem/cilastatin and meropenem. Toxicol Lett 1995;76:23943.

[15] Horiuchi M, Kimura M, Tokumura M, Hasebe N, Arai T, Abe K. Absence of convulsive liability of doripenem, a new carbapenem antibiotic, in comparison with $\beta$-lactam antibiotics. Toxicology 2006;222:114-24. 
Fig. 1. Multivariate Cox regression curves to asses the association with death of the antimicrobial therapies received: meropenem vs. cefotaxime + metronidazole (combined standard chemotherapy) $(P=0.026)$; meropenem vs. imipenem $(P=0.18)$; and imipenem vs. cefotaxime + metronidazole $(P=0.13)$. 


\section{Table 1}

Characteristics of patients with brain abscess in the three therapy groups

\begin{tabular}{|c|c|c|c|c|}
\hline & $\begin{array}{l}\operatorname{CSC}(n= \\
12)\end{array}$ & $\begin{array}{l}\text { Imipenem ( } n \\
=22)\end{array}$ & $\begin{array}{l}\text { Meropenem }(n \\
=25)\end{array}$ & $\begin{array}{l}P- \\
\text { value }\end{array}$ \\
\hline Age (years) (mean \pm & $53.0 \pm$ & $37.5 \pm 19.3$ & $50.0 \pm 15.9$ & 0.016 \\
\hline S.D.) & 14.7 & & & $a$ \\
\hline Gender (male) $[n(\%)]$ & $7(58.3)$ & $18(81.8)$ & $20(80.0)$ & 0.3 \\
\hline GCS score $<7[n(\%)]$ & $3(25.0)$ & $6(27.3)$ & $3(12.0)$ & 0.4 \\
\hline Motor weakness $[n(\%)]$ & $2(16.7)$ & $10(45.5)$ & $10(40.0)$ & 0.2 \\
\hline $\begin{array}{l}\text { Cranial hypertension }[n \\
(\%)]\end{array}$ & $2(16.7)$ & $2(9.1)$ & $4(16.0)$ & 0.7 \\
\hline $\begin{array}{l}\text { Multiple abscesses [ } n \\
(\%)]\end{array}$ & $5(41.7)$ & $7(31.8)$ & $5(20.0)$ & 0.4 \\
\hline No surgery $[n(\%)]$ & $7(58.3)$ & $5(22.7)$ & $4(16.0)$ & $0.02^{b}$ \\
\hline Re-intervention $[n(\%)]$ & $2(16.7)$ & $7(31.8)$ & $3(12.0)$ & 0.5 \\
\hline Death $[n(\%)]$ & $4(33.3)$ & $4(18.2)$ & $1(4.0)$ & $0.06^{c}$ \\
\hline $\begin{array}{l}\text { Days of hospital stay } \\
\text { (mean } \pm \text { S.D.) }\end{array}$ & $\begin{array}{c}31.8 \pm \\
25.4\end{array}$ & $36.6 \pm 20.0$ & $38.8 \pm 24.4$ & 0.7 \\
\hline
\end{tabular}

CSC, combined standard chemotherapy (cefotaxime+ metronidazole); S.D., standard deviation; GCS, Glasgow coma scale.

a Standard therapy versus imipenem $(P=0.048)$ and standard therapy versus meropenem $(P=0.051)$, by Scheffé test.

${ }^{\mathrm{b}}$ Standard therapy versus imipenem $(P=0.06)$ and standard therapy versus meropenem $(P=0.018)$.

${ }^{c}$ Standard therapy versus imipenem $(P=0.4)$ and standard therapy versus meropenem $(P=0.03)$. 
Edited Figure 1

\section{Fig.1.}

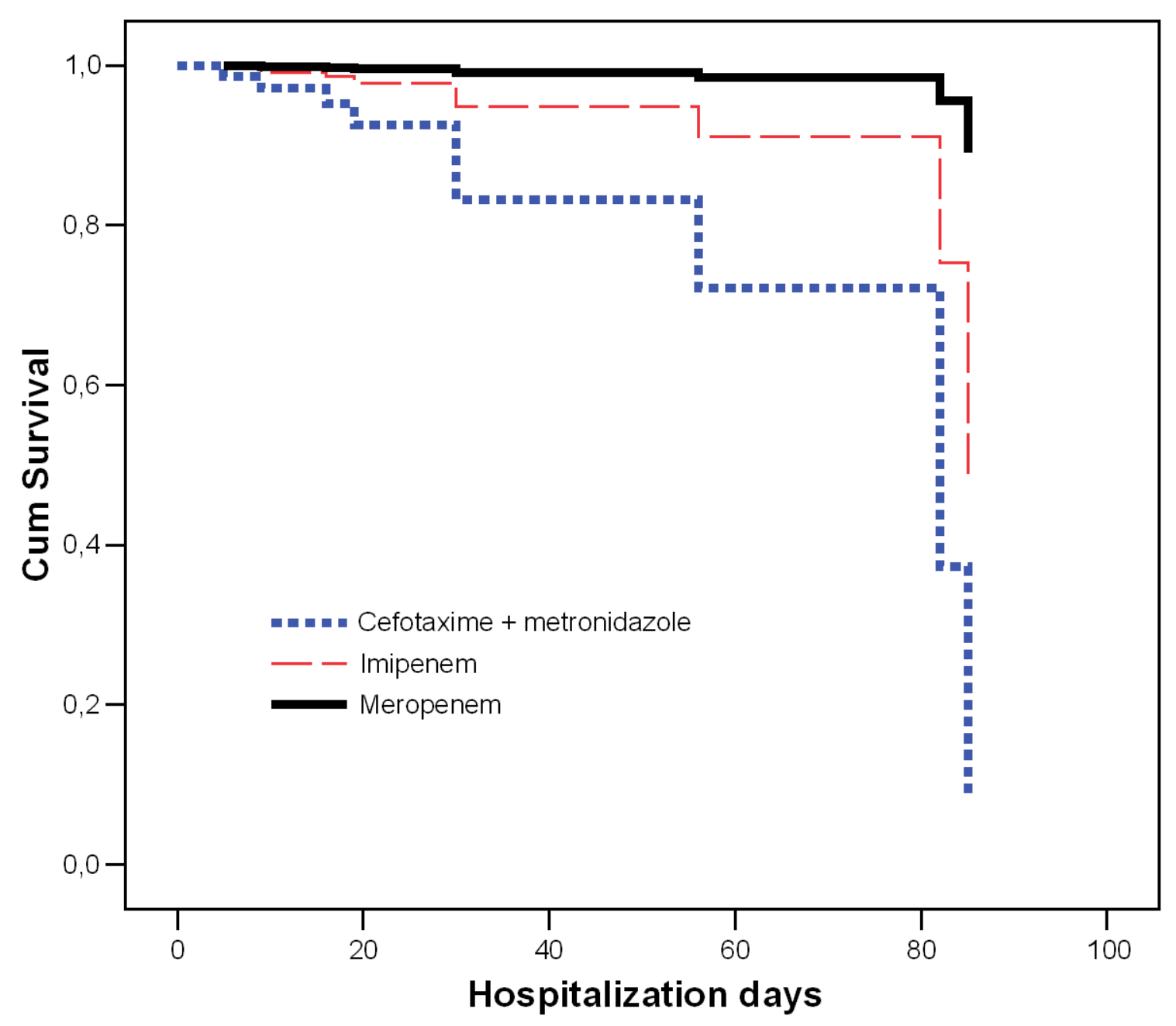

